Journal of Patient-Centered

Volume 3

Issue 4 -- Cardiovascular Aging

Article 11

$11-11-2016$

\title{
Path to Resistance: Risk Factors Associated With Carbapenem- Resistant Pseudomonas aeruginosa
}

Kushal Patel

Jessica J.F. Kram

Dennis J. Baumgardner

Follow this and additional works at: https://aah.org/jpcrr

Part of the Bacterial Infections and Mycoses Commons, Family Medicine Commons, and the Infectious Disease Commons

\section{Recommended Citation}

Patel K, Kram JJ, Baumgardner DJ. Path to resistance: risk factors associated with carbapenem-resistant Pseudomonas aeruginosa. J Patient Cent Res Rev. 2016;3:236.

Published quarterly by Midwest-based health system Advocate Aurora Health and indexed in PubMed Central, the Journal of Patient-Centered Research and Reviews (JPCRR) is an open access, peer-reviewed medical journal focused on disseminating scholarly works devoted to improving patient-centered care practices, health outcomes, and the patient experience. 
$(\mathrm{P}=0.2)$, whereas it continued to show benefit in the group with LVEF of $40 \%-49 \%(\mathrm{P}=0.001)$.

Conclusion: Clinical predictors of mortality in patients with transient LV systolic dysfunction may help further risk-stratify this cohort of patients. It appears that patients with LVEF of $40 \%-49 \%$ continue to derive benefit from ICD therapy.

\section{FIRST PLACE ORAL PRESENTATION}

See page 245 for citation.

\section{SECOND PLACE ORAL PRESENTATION Path to Resistance: Risk Factors Associated With Carbapenem-Resistant Pseudomonas aeruginosa}

\author{
Kushal Patel, Jessica J.F. Kram, Dennis J. Baumgardner
}

Department of Internal Medicine, Aurora Sinai Medical Center; Department of Family Medicine, Aurora UW Medical Group; Center for Urban Population Health

Background: An estimated 51,000 health care-associated Pseudomonas aeruginosa infections occur in the United States annually. More than $13 \%$ are secondary to non-carbapenem multidrug-resistant strains, which result in 400 yearly deaths. Traditional risk factors for resistance include ICU stay, mechanical ventilation, previous hospitalization and major comorbidities. As microbes evolve, risk factors also may evolve.

Purpose: To determine if traditional and/or new risk factors for $P$. aeruginosa resistance are valid and predictive of infection with carbapenem-resistant $P$. aeruginosa.

Methods: We retrospectively studied inpatients and outpatients $\geq 18$ years old who presented to an Aurora Health Care facility with a positive $P$. aeruginosa culture during 2014. Cultures were obtained from the ACL Laboratories database, and patient medical records were reviewed in Epic. Chi-squared test with Yates correction and two-sample t-tests were performed on categorical and continuous variables, respectively. Binary regression was used for multivariable modeling. Significance was associated with $\mathrm{P}<0.05$.

Results: Study population $(\mathrm{N}=1,763)$ characteristics were: mean age 68.0 , body mass index $30.4 \mathrm{~kg} / \mathrm{m}^{2}, 51.2 \%$ female sex, and $89.3 \%$ white race. Resistance to imipenem or meropenem $(14.0 \%)$ on univariable analysis was associated with younger age ( 66.0 vs 68.3 years, $\mathrm{P}=0.027)$, hospitalized patients $(19.7 \%$ vs $8.6 \%, \mathrm{P}<0.0001)$, male sex $(16.0 \%$ vs $12.0 \%, \mathrm{P}=0.017)$, nonwhite race $(23.5 \%$ vs $12.3 \%, \mathrm{P}<0.0001)$, respiratory culture $(30.9 \%$ vs $12.1 \%, \mathrm{P}<0.0001)$, history of pulmonary disease $(19.4 \%$ vs $12.9 \%, \mathrm{P}=0.005)$, history of congestive heart failure ( $18.6 \%$ vs $13.0 \%, \mathrm{P}=0.016)$, history of multidrug resistance $(33.3 \%$ vs $13.6 \%, \mathrm{P}=0.003)$ and recent surgery $(17.8 \%$ vs $12.2 \%, \mathrm{P}=0.002)$, as well as transfer from institution, Foley catheter, vasopressor treatment, central/PIC lines, mechanical ventilation, ICU admission, and bedridden status (all $\mathrm{P}<0.0001$ ). In multivariable modeling, nonwhite race, respiratory culture, recent transfer, vasopressor use and central/PIC lines were significant. Only $0.57 \%$ of strains were resistant to the six traditional non-carbapenem drugs and both carbapenems.

Conclusion: Demographic and traditional risk factors, as well as respiratory cultures, were predictive of carbapenem resistance. Such information may guide initial antibiotic treatment of $P$. aeruginosa. Fortunately, less than $1 \%$ of strains were resistant to all drugs tested. Further studies looking at change in outcome while incorporating these risk factors in determination of empiric coverage for patients should be performed.

\section{THIRD PLACE ORAL PRESENTATION}

See page 245 for citation.

\section{FIRST PLACE POSTER}

See page 245 for citation.

\section{SECOND PLACE POSTER (tie)}

The Association Between Doppler Measures of Cardiac Function and Outcomes in Patients With Left Ventricular Ejection Fraction $\leq \mathbf{4 0} \%$ Undergoing Noncardiovascular Surgeries

Yang Shi, Rachel Pedersen, Matthew Rappelt, Robyn Shearer, Nasir Z. Sulemanjee, Dianne L. Zwicke, T. Edward Hastings, Omar M. Cheema, Vinay Thohan

Sheikh Khalifa bin Hamad Al Thani Center for Integrative Research on Cardiovascular Aging, Aurora Research Institute; Transplant Administration, Aurora Health Care; Aurora Cardiovascular Services, Aurora Health Care

Background: Preoperative risk assessments of individuals who undergo major noncardiac surgery have focused on ischemic heart disease. Information on how to assess the noncardiac surgical risks for patients with depressed cardiac function, as seen in heart failure, is sparse. Echocardiography is routinely performed in patients with depressed cardiac function and is an accepted standard cardiac assessment. Transthoracic echocardiography (TTE) provides strong independent prognostic implications in a wide range of cardiovascular conditions.

Purpose: To identify the echocardiographic parameters associated with outcomes among patients undergoing major noncardiac surgery.

Methods: A retrospective single-institution investigation identified 1,770 patients who underwent one or more major noncardiac procedures from Jan. 1, 2011, to June 30, 2014, and had at least one TTE performed within 90 days before surgery. Patients were stratified by presurgery left ventricular ejection fraction (LVEF) into LVEF $\leq 40 \%$ and LVEF $>40 \%$ groups. The cohort was followed through June 12, 2015, with the outcome focused on all-cause mortality. Continuous and categorical variables were compared by Student's t-test and chi-squared test, respectively. Kaplan-Meier method was used to calculate mortality estimates postsurgery. Cox proportional hazards model was used for univariate and multivariable models. 